\title{
Sensory Imagery in the Context of Beverage Advertising: How the Senses Affect Product Design and Attitude: An Abstract
}

\author{
Klaus-Peter Wiedmann, Janina Haase, and Jannick Bettels
}

\begin{abstract}
Research in sensory marketing provides evidence for the great potential of sensory imagery to create sensory consumer experiences. Especially in the context of food and beverage advertising, the targeted appeal of the senses through sensory imagery seems to be promising. However, there are still some research gaps relating to the concrete effect sizes of sensory appeals and possible mediators such as product design. This paper aims at closing these gaps by focusing on two different research issues. First, it investigates the effects of sensory imagery on marketingrelated key performance indicators (i.e., sensory perception, product design, and attitude) by using analysis of variance. Further, the paper examines underlying causal relationships between these potential market success factors by applying PLS-SEM. The findings support the usefulness of sensory imagery in advertisements, as it appears to be a valuable approach to address specific senses and to have a positive impact on consumer perception. Moreover, the results reveal a causal chain of several direct and indirect effects between relevant performance indicators. Implications for marketing managers can be derived from this research on how to design powerful advertisements and effectively appeal to all five human senses by relying on sensory imagery.
\end{abstract}

References Available Upon Request

K.-P. Wiedmann · J. Haase $(\bowtie) \cdot J$. Bettels

Leibniz University of Hannover, Hannover, Germany

e-mail:wiedmann@m2.uni-hannover.de; haase@m2.uni-hannover.de;

bettels@m2.uni-hannover.de 